\title{
Actitud emprendedora y estilos emocionales. Contribuciones para el diseño de la formación de futuros emprendedores
}

Entrepreneurial attitude and emotional styles. Contributions to the design of the training of future entrepreneurs

\author{
Emilse Durán-Aponte \\ Universidad Simón Bolívar \\ Camurí Grande, Venezuela \\ emilseaponte@usb.ve
}

Diana Arias-Gómez²

Universidad Simón Bolívar

Camurí Grande, Venezuela

darias@usb.ve

Recibido 16 julio 2015 • Aceptado 03 junio 2016 • Corregido 10 junio 2016

Resumen. El emprendedor es un innovador, un estratega, un creativo y alguien que cree en sus ideas para llevarlas a cabo de forma exitosa. Sin embargo, algunos factores de tipo emocional llegan a interponerse entre la actitud del emprendedor y finalmente el acto de emprender, los cuales podrían ser vitales para el diseño de la formación de futuros emprendedores. El objetivo de este trabajo fue analizar la relación que existe entre la actitud emprendedora de un individuo y los estilos emocionales que posee. Participaron 259 estudiantes venezolanos, de los cuales 94 eran de la Universidad Central de Venezuela, inscritos en las carreras de Administración y Contaduría, y 165 de la Universidad Simón Bolívar, cursantes de ingenierías y carreras cortas administrativas e industriales, a través de una metodología descriptivo-correlacional, con un diseño no experimental. Se utilizó la escala unidimensional ¿Soy del tipo emprendedor? (STE) para medir la actitud emprendedora y el Cuestionario de Estilo Emocional (CEE). Con un 33\% de varianza explicada, los resultados indican que una persona con alta actitud hacia el emprendimiento tiene una relación negativa con el estilo

1 Doctora en Ciencias Sociales y Humanidades, Universidad Simón Bolívar (USB). Magíster en Psicología, USB. Licenciada en Educación, Universidad Simón Rodríguez. Profesora a dedicación exclusiva de la USB. Coordinadora del Laboratorio de Investigación en Bienestar y Rendimiento Estudiantil (LIBRE) www.libre.usb.sl.ve. Líneas de investigación: procesos de enseñanza y aprendizaje, vida estudiantil y formación universitaria. Autora de más de 20 artículos de investigación, entre ellos: Aporte de variables cognitivo- motivacionales en la implementación de un foro electrónico: caso asignatura termodinámica. Revista Didáctica, Innovación y Multimedia, 29-2014, 1 - 9 y Validación del Inventario de Autoeficacia para Inteligencias Múltiples Revisado (IAMI-R) en una muestra de estudiantes universitarios venezolanos, Actualidades Investigativas en Educación, 14-2014, 1 - 23. Responsable de cinco proyectos de investigación avalados y financiados por el Decanato de Investigación y Desarrollo, Universidad Simón Bolívar, en este momento ejecutándose: Aplicación Web para la detección del riesgo académico en estudiantes que cursan el Ciclo de Iniciación Universitaria y perfil vocacional de aspirantes a ingresar al sistema universitario venezolano.

2 Tesista de la maestría en Educación mención Procesos de Aprendizaje, Universidad Católica Andrés Bello (UCAB). Especialista en Dinámica de Grupos, Universidad Central de Venezuela. Licenciada en Educación mención Ciencias Pedagógicas, UCAB. Profesora a tiempo integral de la USB. Jefa de sección del área de Desarrollo de Destrezas Intelectuales. Responsable de los proyectos de investigación: Programa de capacitación en competencias para el trabajo efectivo en equipos dentro de un ambiente académico a los estudiantes que cursan el CIU, Universidad Simón Bolívar, en la Sede del Litoral y Orientación a las metas académicas, persistencia y rendimiento académico en estudiantes del Ciclo de Iniciación Universitaria, ambos avalados y financiados por el Decanato de Investigación y Desarrollo, Universidad Simón Bolívar. 
emocional rumiación. En específico, la proactividad se relaciona de forma negativa con la inhibición y la rumiación, y el optimismo solo con la rumiación. Entre las orientaciones para el diseño de planes de formación deberá considerarse el ejecutar un diagnóstico de las características del futuro emprendedor, incorporar de forma trasversal el desarrollo de la actitud emprendedora en cuanto a optimismo y proactividad, además de enseñar el manejo y control de las emociones.

Palabras clave. Emociones; emprendimiento; universitarios; educación

Abstract. An entrepreneur is an innovator who is also creative and a strategist - someone who firmly believes in their own ideas and executes the successfully. Yet, certain emotional factors stand out among entrepreneurs when undertaking or executing plans, which could be critical for designing future entrepreneurial training programs.

This article shows the results of a study in Venezuela that analyzed the relationship among entrepreneurial attitudes and emotional styles as observed in the students who participated, 94 of which were from Central University of Venezuela majoring in Business Administration and Accounting, and 165 students from the Simon Bolivar University majoring in Engineering and shortterm Administrative and Industrial career paths.

A descriptive, correlational, non experimental design was used based on the "Am I an Entrepreneurial Type?" (STE) scale to measure entrepreneurship as well as an Emotional Style Questionnaire (EEC). The results showed a $33 \%$ variance, indicating that an individual with a strong attitude towards entrepreneurship showed negative emotional rumination styles. They are proactive onl yin terms of their propensity towards rumination. Given these results, training guidelines should include addressing the traits of the future entrepreneur and a cross-cutting inclusion of optimism and proactive entrepreneurial attitudes as well as how to control and manage emotions.

Keywords. Emotions; entrepreneurship; university education.

\section{Introducción}

En la revisión de estudios relacionados con las características claves en el perfil de un futuro emprendedor, es común encontrar la presencia de rasgos psicológicos asociados a la persona que emprende (Espíritu, 2011), lo que posibilita, en gran medida, identificar aquellos con altas intenciones de desarrollarse como emprendedor y los que no. Marulanda, Correa y Mejía (2009) opinan que las características psicológicas del emprendedor hacen posible inferir que no existe un perfil único para los emprendedores, sino que la combinación de características de tipo cultural, económico y personal influye más en algunas personas que en otras y por ende, solo algunos se involucran en el emprendimiento.

Una integración entre la psicología y modelos de gestión aplicables al emprendimiento, ha dado como resultado la aparición de aportes valiosos a través de enfoques teóricos que permiten explorar los rasgos de un futuro emprendedor, entre ellos están: el Modelo sobre el Evento Emprendedor de Shapero y Sokol (1982), adaptado por Sánchez, Lanero y Yurrebaso (2005); la aplicación de la Teoría del Comportamiento Planificado, adaptada por Flores y Barberá 
(2013) y la Teoría de la Conservación de Recursos, adaptado por Salazar-Carvajal, HerreraSánchez, Rueda-Méndez y León-Rubio (2014) a la explicación del emprendimiento. Todos ellos coinciden en afirmar que existen determinadas competencias, aptitudes y habilidades personales que condicionan la decisión de emprender.

De todos ellos se desprenden recomendaciones para la formación de un futuro emprendedor; sin embargo, la mayoría se concentra en asuntos contextuales, dejando de lado las características personales y sobre todo emocionales de la persona que va a emprender. Expresiones como las de Alvarado y Rivera (2011) que afirman que un número más elevado de asignaturas y actividades académicas relativas a los negocios fortalecen el deseo o intención de formar en el futuro una propia empresa, dan cuenta del énfasis que hasta ahora se ha dado en las universidades a la formación gerencial hacia el emprendimiento, por encima de los aspectos psicológicos para ello.

No obstante, si se ve el emprendimiento como una posibilidad de riesgo de atreverse a incursionar en algo, para algunos puede resultar una aventura desconocida; es necesario entonces buscar elementos que contribuyan con un programa de formación emprendedora más acorde con lo humano del individuo, que impacten en áreas relacionadas con la actitud y las emociones.

Esta investigación corresponde a una segunda etapa del proyecto denominado“Formación universitaria para el emprendimiento", desarrollado por el Laboratorio de Investigación en Bienestar y Rendimiento Estudiantil (Libre). En principio, Durán-Aponte y Arias-Gómez (2015) analizaron la existencia o ausencia de relación entre la intención emprendedora y un conjunto de variables cognitivas y sociopersonales, de donde se desprendió la necesidad de profundizar en la relación de los aspectos psicológicos del individuo, al encontrar relaciones significativas entre la actitud de emprender y sus estilos emociones, las cuales no fueron analizadas en su momento, pues no eran el objeto de dicho estudio.

\section{Orientaciones para la formación emprendedora}

Arroyo-Vázquez y Jiménez-Sáez (2008) recomiendan que la enseñanza sobre innovación y emprendimiento en las universidades, no se debe limitar a la creación de empresas y a una formación esencialmente empresarial, sino que implica una cultura emprendedora en todos los ámbitos, no solo el gerencial sino en el nivel personal y psicológico. Del mismo modo, la reciente investigación de Mata, Medina, Martínez, Alcocer, Castillo y Vázquez (2015) coincide con las reflexiones de Merino y Vargas (2011) y Martínez y Carmona (2009), cuando afirman que es necesaria una formación integral para el individuo orientado hacia el emprendimiento. Mata et al. (2015) encontraron que los estudiantes universitarios valoran como competencias emprendedoras aquellas relacionadas con liderazgo, comunicación, planificación/organización y decisión. Estas competencias deben complementar la formación en planes de negocio que 
dominan actualmente los programas universitarios sobre el emprendimiento. Como lo afirman Merino y Vargas (2011), en todo plan formativo para el emprendimiento se debe considerar la interacción tanto de niveles individuales o psicológicos como institucionales y del entorno, pues todos influyen en la decisión de emprender.

Entre las características psicológicas del futuro emprendedor, reconocidas en diferentes investigaciones, se tienen: la autoeficacia emprendedora (Salvador, 2008; Sánchez, 2010; Moriano, Topa, Morelo, Entenza \& Lévy-Mangin, 2012; Durán-Aponte, 2013a), la actitud emprendedora (Roth \& Lacoa, 2009; Loli, Del Carpio \& La Jara, 2009; Espíritu, 2011) y los estilos o personalidad emocional (Salvador, 2008; Durán-Aponte, 2013b; Bernal, 2014). Específicamente, la actitud emprendedora y los estilos emocionales ocupan especial interés en este estudio, debido a que ambos factores han sido considerados en otras investigaciones como elementos que pueden contribuir u obstaculizar la intención de emprender.

Estas recomendaciones requieren, entonces, de un marco conceptual basado en evidencia empírica que justifique el abordaje de la actitud y las emociones en los futuros emprendedores. Para ello, será necesario conocer en qué sentido se da la relación entre estas variables y analizar las posibles razones por las que esto sucede, de manera que cada institución universitaria pueda gestionar un plan acorde con las necesidades reales del individuo.

\section{Actitud emprendedora}

En el trabajo desarrollado por Alda-Varas, Villardón-Gallego y Elexpuro-Albizuri (2012) se plantea la necesidad de presentar un perfil como herramienta fundamental para el diseño de la formación de emprendedores, pues esto facilita el establecimiento de metas de formación. Entre las características de dicho perfil, los autores recomiendan desarrollar autonomía, autoconfianza, asertividad, creatividad, optimismo, confianza, entre otros. Estas características son distintivas de la actitud emprendedora y, aunque tradicionalmente los estudios relacionados con la identificación del perfil emprendedor utilizan de forma indistinta los constructos autoeficacia y actitud emprendedora, la reciente investigación de Durán-Aponte (2013b) resulta un intento exitoso para diferenciar entre ambos constructos, a través de un estudio de validez de constructo. La autora afirma que la autoeficacia emprendedora se refiere al juicio que hacen las personas sobre su capacidad de emprender un negocio, en cambio la actitud emprendedora considera que la persona deberá ser optimista, proactiva, persistente y creativa, lo cual está más relacionado con su personalidad.

De forma específica, Durán-Aponte (2013b) define las dimensiones de la actitud emprendedora de la siguiente manera:

- Optimismo: es la respuesta positiva ante adversidades relacionadas con el negocio, en donde se tiene una alta confianza de lograr proyectos exitosos, sortear problemas sin miedo y con disposición a emprender ideas nuevas. 
- Proactividad: es la exigencia autoimpuesta por el individuo para hacer de la mejor forma posible todo lo que emprende, involucra retarse, apuntar a la excelencia y dar lo mejor de sí mismo.

- Persistencia: comprende el reconocimiento de los errores propios, aprender de estos y la persistencia para sobreponerse e intentar nuevamente, por lo cual no abandona la actividad emprendedora cuando tiene situaciones adversas, sino que busca soluciones alternativas.

- Creatividad e innovación: se trata de una actitud imaginativa y creativa que facilita el funcionamiento de las cosas, la satisfacción de necesidades y la búsqueda de respuestas nuevas a problemáticas planteadas.

Duran-Aponte (2013b) afirma que un individuo con actitud emprendedora no copia iniciativas de emprendimientos, sino que se esfuerza por desarrollar y crear cosas nuevas, o innovar sobre lo que ya existe con una alta persistencia hasta lograrlo.

Como antecedente, la investigación de Martínez y Carmona (2009) destacó que entre las competencias a desarrollar en la formación emprendedora relacionadas con la actitud, están:

- Actitud mental positiva: esto significa hacer que los sujetos lleguen a confiar en sus propias capacidades y habilidades y, de este modo, podrán desarrollar con éxito su propia empresa.

- Capacidad para sobreponerse al fracaso: se trata de motivarlos y formarlos para que no abandonen el proyecto empresarial que emprendan, a pesar de dificultades o adversidades.

- Facilidad para las relaciones sociales: los emprendedores deberán aprender a relacionarse con facilidad y de manera adecuada con otras personas, en diferentes situaciones y contextos.

- Iniciativa: Consiste en tener una actitud proactiva, es decir, hacer cosas por sí mismos, promover iniciativas, introducir nuevos productos, servicios o tecnología innovadores.

- Visión y proyecto de futuro: generar ideas e identificar las oportunidades que no han sido vistas por otros empresarios.

Al mismo tiempo, la investigación de Loli et ál. (2009) revela que algunas dimensiones como la perseverancia, la creatividad y el optimismo se dan en altos puntajes en aquellos con intenciones de emprender un negocio. Específicamente en cuanto a la creatividad, Orrego $(2008$, p. 230) plantea que "todo emprendedor tiene capacidad de innovar y generar 
ideas diferentes que mejoren su entorno y por ende su nivel de vida"; por lo tanto, es una característica fundamental en quien se considera un futuro emprendedor. Para el caso de la persistencia, Cordero, Astudillo, Delgado, Amón y Carpio (2011) afirman que de tres conductas emprendedoras que inciden de manera positiva en el emprendimiento, la persistencia es una de ellas, la cual debe estar en el primer lugar de las cualidades que valore un emprendedor, antes que fijarse metas y ser eficiente.

Roth y Lacoa (2009) indican que cuando se reconoce a una persona con potencial emprendedor o con alta actitud hacia el emprendimiento, se le relaciona con condiciones y afinidades para el cambio y la innovación, así como una clara capacidad para renovarse ante la adversidad y una serie de convicciones acerca de sus posibilidades de ejercer control sobre su conducta emocional. Como en todo constructo psicológico, se debe tener en cuenta que poseer o no una alta actitud emprendedora dependerá de otros factores personales que pueden ser subjetivos o sociales y que tienen un efecto positivo o negativo en la forma que aborda un individuo la posibilidad de emprender. Uno de estos factores es la mencionada inteligencia emocional, pues se cree que esta condiciona la conducta del que tiene la intención de emprender a través de los estilos emocionales que el individuo manifieste (Bernal, 2014). En este caso, el control sobre la conducta emocional que pueden tener las personas con actitud emprendedora debe darse de manera que potencie el emprendimiento, por lo tanto, es importante profundizar en este aspecto.

\section{Estilos emocionales}

Los resultados de Roth y Lacoa (2009, p. 31) sugieren que "todo proceso de emprendimiento puede conducir a cierto desgaste emocional, que debe ser adecuadamente controlado para evitar que se interponga entre la intención de emprender y el emprendimiento mismo". Este posible desgaste viene dado por las mismas presiones a las que se expone el individuo, en palabras de Salazar-Carvajal et al. (2014), la incertidumbre en el emprendimiento puede afectar a las personas en su salud y bienestar psicológico provocando entre otras cosas, estrés, ira o ansiedad.

Se trata entonces, de entender qué contribuye a que algunos individuos puedan afrontar estos eventos mejor que otros; de allí, que cobre valor la idea de la inteligencia emocional. Este término surge primeramente, de las afirmaciones de cuando se señalaba que la inteligencia intrapersonal e interpersonal, que formaban parte de su modelo teórico de las Inteligencias Múltiples, podían ser integradas en un solo constructo reconocido como inteligencia personal (Gardner, 1995;Gardner, 2000).Para este autor, esta inteligencia involucraba evaluary comprender emociones, y también la habilidad para regularlas frente a los demás. Posteriormente, pasaron a estar presentes en los postulados de Goleman (1995), denominándose inteligencia emocional.

Según lo reseña Salvador (2008), la inteligencia emocional ha sido abordada a través de diversos modelos teóricos entre los que están: el Modelo de Habilidad de Mayer y Salovey (1997) y el Modelo de Competencias Emocionales de Goleman (1998). Estos modelos tienen en 
cuenta el grado en que los individuos pueden identificar correctamente sus propias emociones, sentimientos y necesidades como los de los demás, la capacidad para estar abiertos a los estados emocionales positivos y negativos, así como la habilidad para reflexionar sobre las emociones y determinar la utilidad de la información.

Posteriormente Mayer, Caruso y Salovey (1999) plantean el Modelo de Habilidad sobre la Inteligencia, en el que se asume que los seres humanos procesan información y esto les permite responder de manera adaptativa ante el entorno. Así, algunas personas responden mejor a los estímulos y experiencias del medio que otras, y se les concibe como inteligentes emocionales, pues perciben, de manera exacta, la información de las emociones propias y de los demás, y aprenden a manejar sus emociones y las de otros individuos.

Otra forma de ver la inteligencia emocional se da cuando se toman en cuenta la capacidad o ausencia de esta para manejar las emociones. En este sentido, un modelo que ofrece una explicación para aquellos individuos con dificultad para comprender y expresar sus emociones y las de los demás, es el de los Estilos Emocionales. Guarino (2011) expone dos estilos emocionales para explicar mejor esta situación; por un lado, la Inhibición emocional que se asocia con el temor al fracaso y a ser expuesto ante otros, y la Rumiación, relacionado con el miedo a enfrentar el futuro. La autora los resume así:

- Inhibición emocional: se refiere a abstenerse o al "embotellamiento" de la expresión de las emociones experimentadas. Esto también incluye el miedo a solicitar ayuda y a la vez, ser expuesto ante otros.

- Rumiación: se refiere a la propensión de los individuos a preocuparse por eventos molestos que ocurrieron en el pasado. Esto también incluye poca confianza en las personas, dificultad para superar los fracasos y miedo a enfrentar el futuro.

La Inhibición emocional, se considera un estilo de respuesta de aquellas personas que tienen inconvenientes para comprender y expresar tanto las emociones propias como las ajenas, lo que según Guarino (2011) significa que la persona se inhibe emocionalmente, dejando que estas emociones definan su conducta. En el caso de la rumiación, los individuos con carencia de habilidades sociales e interpersonales evidencian dificultades para expresar sus propias emociones, los cuales, generalmente, también manifiestan una alta tendencia a evitar las expresiones emocionales de los otros.

\section{Actitud emprendedora y estilos emocionales}

Se cree que estos estilos emocionales influencian la actitud emprendedora debido a que se pueden convertir en obstáculos para la proactividad y el optimismo. Pihkala y Vesalainen (2000) afirman que, entre las barreras inhibidoras que deben superar los emprendedores están 
el riesgo social o miedo a hacer el ridículo si la idea de negocio resulta un fracaso y el miedo a ser visto por otros como un perdedor o fracasado. Moriano, Palací y Morales (2006) advierten que cuanta más importancia le dé la persona a estas barreras, menor será la intención de desarrollar su carrera profesional a través del autoempleo. Del mismo modo, Brown y Marshall (2001), citado por López \& García (2011), cuando el individuo tiene expectativas positivas, esto influye en la realización de tareas difíciles porque existe más entusiasmo y vigor para enfrentar los desafíos.

La investigación de Salvador (2008) mide la inteligencia emocional en cuatro dimensiones (claridad, atención, manejo y utilización) y la relaciona con la autoeficacia emprendedora (desarrollo de productos y gestión de recursos humanos); de allí, se extrae que la claridad emocional y utilización de las emociones son predictores eficaces del desarrollo de nuevos productos y el aprovechamiento de oportunidades de mercado, lo cual está relacionado con la creatividad e innovación, por lo tanto, si un individuo presenta altos niveles de inhibición emocional y rumiación, se afectará negativamente la actitud hacia el emprendimiento.

Salvador (2009) continuando con su línea de investigación, determinó que independientemente de la edad, el sexo y el contexto sociocultural, el empleo de las emociones es la variable que siempre desprende relaciones más elevadas con el emprendimiento. Así, teniendo en cuenta que los estilos emocionales abordados en esta investigación evalúan el grado en que el individuo es incapaz de manejar sus emociones, sea que no logre expresarlas o que no pueda olvidar los sentimientos negativos o de fracaso, un individuo que posea altos puntajes en ellos tendrá dificultades para enfrentar los retos inesperados de los negocios, principalmente en medio de ambientes de alta competencia.

En función de esto, el objetivo del presente estudio es analizar la relación que existe entre la actitud emprendedora de un individuo y los estilos emocionales que posee, lo cual puede ser vital para entender de qué manera contribuye en la formación emprendedora.

\section{Método}

La metodología empleada fue de tipo descriptivo-correlacional, integrada con un diseño no experimental (Kerlinger \& Lee, 2002) y transversal en el que se recolectaron los datos en un solo momento. Participó una muestra de 259 estudiantes, de los cuales 94 eran de la Universidad Central de Venezuela, inscritos en las carreras de Administración y Contaduría, y 165 de la Universidad Simón Bolívar, cursantes de ingenierías y carreras cortas administrativas e industriales. De los participantes, 119 eran hombres y 140 mujeres, con edades entre 17 y 27 años, con una media de 20,22 y una DT de 2,110.

Para medir la variable actitud emprendedora se aplicó la escala ¿Soy del tipo emprendedor? (STE) original de Gassé (1983), citado por Roth \& Lacoa, 2009), la cual fue adaptada para este estudio por Durán-Aponte (2013b). El instrumento contiene 15 ítems que se miden en una escala tipo Likert con puntuaciones que van del uno al cuatro (uno totalmente en desacuerdo 
al cuatro totalmente de acuerdo). La confiabilidad fue de 0,845 para la escala total y consta de cuatro dimensiones denominadas: optimismo, proactividad, persistencia y creatividad.

En el caso de los estilos emocionales, se utilizó el Cuestionario de Estilo Emocional (CEE), el cual fue adaptado y validado por Guarino (2011) para su uso en población de habla hispana. Este se compone de 39 ítems que se miden con dos opciones: cierto (C) o falso (F). El alfa para la escala total fue de 0,865, y consta de dos estilos denominados: inhibición emocional y rumiación.

Se explicó a los participantes sobre el objetivo de la investigación y se les indicó que se solicitaba su participación de manera voluntaria, la cual aceptaron firmando un consentimiento para procesar sus respuestas; estas serían tratadas de forma estrictamente confidencial. Posteriormente, se aplicaron los cuestionarios durante las sesiones de clase, con una duración aproximada de 10 minutos Los datos se transcribieron y procesaron utilizando el paquete estadístico SPSS v.19y se realizaron los análisis estadísticos descriptivos, a través de correlaciones y multivariantes con el análisis de regresión múltiple. Finalmente, se analizan los datos y se generan las conclusiones.

Las hipótesis que orientan esta investigación son las siguientes:

H.1. Los estilos emocionales rumiación e inhibición emocional se relacionan negativamente con las dimensiones de la actitud emprendedora.

H.2. El estilo emocional rumiación tiene mayor relación negativa con las dimensiones de la actitud emprendedora que la inhibición emocional.

H.3. La actitud emprendedora es mayor cuando los estilos emocionales rumiación e inhibición son menores.

\section{Resultados}

En la Tabla 1 se muestran los descriptivos para las variables del estudio y se observa que, de las dimensiones de la actitud emprendedora, la variable persistencia muestra una tendencia moderada alta $(M=3,59)$ y le siguen el resto de las dimensiones de la variable. En cuanto a los estilos emocionales, la inhibición emocional presenta el mayor promedio de forma moderada baja $(M=9,95)$, respecto al máximo, e igualmente sucede con la rumiación. 
Tabla 1

Estadísticos descriptivos de las variables

\begin{tabular}{|l|c|c|c|c|}
\hline Descriptivos & Media & DT & Mínimo & Máximo \\
\hline Optimismo & 3,27 &, 505 & 1,50 & 4 \\
\hline Proactividad & 3,47 &, 479 & 1 & 4 \\
\hline Persistencia & 3,59 &, 461 & 1,50 & 4 \\
\hline Creatividad & 3,22 &, 554 & 1 & 4 \\
\hline Inhibición & 9,95 & 2,15 & 4 & 15 \\
\hline Rumiación & 7,02 & 3,66 & 1 & 19 \\
\hline
\end{tabular}

Nota: Elaboración propia con base en la información suministrada por los participantes, 2015.

La Tabla 2 muestra las relaciones bivariadas entre las dimensiones de actitud emprendedora y los estilos emocionales, en donde la Rumiación correlaciona de forma negativa con la dimensión de actitud emprendedora denominada Optimismo $(r=-, 238 ; \mathrm{p}<, 000)$, y con la dimensión Proactividad ( $r=-, 199 ; \mathrm{p}<, 005)$. El estilo emocional Inhibición correlaciona de forma negativa con la dimensión Proactividad $(r=-, 173 ; p<, 005)$.

\section{Tabla 2}

\section{Correlaciones entre las variables}

\begin{tabular}{|l|l|l|l|l|l|}
\hline Variables & Proactividad & Persistencia & Creatividad & Inhibición & Rumiación \\
\hline Optimismo &, $551^{* *}$ &, $447^{* *}$ &, $470^{* *}$ &,- 105 &,$- 238^{* *}$ \\
\hline Proactividad & 1 &, $473^{* *}$ &, $464^{* *}$ &,$- \mathbf{1 7 3 *}$ &,$- \mathbf{1 9 9 *}$ \\
\hline Persistencia & & 1 &, $447^{* *}$ &,- 028 &,- 141 \\
\hline Creatividad & & & 1 &,- 047 &,- 073 \\
\hline Inhibición & & & & 1 &, $\mathbf{1 7 6 *}$ \\
\hline Rumiación & & & & & 1 \\
\hline
\end{tabular}

Nota: Elaboración propia con base en la información suministrada por los participantes, 2015.

En cuanto a la capacidad predictiva de los estilos emocionales sobre la sumatoria de los puntajes de todas las dimensiones de la actitud emprendedora, que se obtuvo mediante la aplicación de un análisis de regresión múltiple, se consigue un 33\% de varianza explicada (R2= 
$0,33, \mathrm{sig}=0,021)$. De las dos dimensiones introducidas en el modelo, se observa en la Tabla 3, que solo resultó significativo el estilo emocional rumiación (Beta= -0,341, sig.=0,015).

Tabla 3

Resumen del modelo de regresión lineal múltiple

\begin{tabular}{c|c|c|c|c|c|c}
\hline$R$ & $R^{2}$ & Error & Sig. & Beta & Sig. & t \\
\hline \multirow{2}{*}{0,231} & \multirow{2}{*}{$\mathbf{0 , 3 3}$} & \multirow{2}{*}{0,677} & \multirow{2}{*}{0,021} & Rumiación & $\mathbf{- 0 , 3 4 1 ^ { * }}$ & 0,121 \\
\cline { 5 - 7 } & & & Inhibición & $-0,181$ & 0,015 \\
\hline
\end{tabular}

Nota: Elaboración propia con base en la información suministrada por los participantes, 2015.

\section{Discusión de resultados}

Retomando las hipótesis planteadas en la investigación, basadas en las relaciones entre las dimensiones de la actitud emprendedora y los estilos emocionales, se tiene que la primera hipótesis es confirmada parcialmente, debido a que los estilos emocionales rumiación e inhibición emocional se relacionan negativamente con solo dos dimensiones de la actitud emprendedora. La relación negativa entre el optimismo y la rumiación es consistente con los planteamientos de Roth y Lacoa (2009), y permiten sugerir que cuando no se superan dificultades del pasado, el individuo está inhabilitado para emprender ideas nuevas, su miedo y desconfianza le impiden tener una respuesta positiva ante adversidades que se relacionen con el negocio. En palabras de López y García (2011), se estaría frente a una situación de pesimismo, lo cual afectará notablemente la actitud de emprendedor.

En cambio, según González y Valdez (2012), al ser mayor la orientación optimista, los individuos experimentan más sentimientos positivos en caso de dificultades y esto ayuda a la actitud de emprender. Según estos autores, el pesimismo hace desistir ante los retos y por ello, la experiencia de fracaso se torna más frecuente, aun cuando el éxito fuera posible con tan solo un poco de esfuerzo y persistencia. De esta manera, se piensa que el optimismo es un factor protector frente a la adversidad.

A esto, se suma la relación negativa entre la proactividad y la rumiación, donde es posible interpretar que, tal como lo indica Durán-Aponte (2013b), la proactividad tiene que ver con la necesidad de lograr aquello que se emprende, en cambio la rumiación ve el futuro con pocas expectativas, maximiza lo negativo y los fracasos, por ello el individuo se puede tornar reactivo, lo que hace muy difícil autoexigirse y ponerse retos, pues se duda de tener éxito en ellos. Esto es consistente con lo dicho por Moriano et al. (2006), quienes vieron el miedo al fracaso como una barrera para desarrollarse como emprendedor. No se trata de que el emprendedor no tenga miedo, la diferencia radica en que sabe qué hacer con él. 
Para Sánchez, Caggiano y Hernández (2011), una persona con personalidad proactiva no necesariamente se percibe como más propenso a asumir riesgos, sino que se refiere a la tendencia a iniciar y mantener acciones que alteran el ambiente que les rodea. En este sentido, cuando se tenga mayor proactividad estará en mejor capacidad para categorizar las situaciones de riesgo como más positivas, alejando los sentimientos de derrota y fracaso del pasado, y los posibles que ocurran en el presente y futuro.

De modo similar ocurre con la relación negativa entre la proactividad y el estilo emocional inhibición, aunque es una relación poco encontrada en estudios previos, es posible entender que los individuos con dificultades para expresar las emociones propias, incluyendo el miedo a pedir ayuda y a ser expuesto ante otros, tendrán problemas a la hora de iniciar e impulsar metas y planes empresariales, pues la desconfianza en los demás y en sus propias capacidades les impedirá pensarse eficaces de dirigir sus propios empleados o supervisar personal. Junto a esto, el deseo de no mostrar lo que piensan o sienten, les inhibe a dar lo mejor de sí mismos en cuanto al negocio a emprender. Debe tener en cuenta, el futuro emprendedor, que si las palabras de otros o lo que piensen de él pueden hacerle cambiar de opinión, entonces debe buscar una forma de desarrollar una personalidad más fuerte para estar en capacidad de afrontar esta responsabilidad.

El resto de las dimensiones de la actitud emprendedora (creatividad y persistencia), no tuvieron relación con los estilos emocionales. Se puede entender que la creatividad no esté relacionada directamente con el manejo de las emociones, pues esta es una característica imprescindible para quien desea emprender $y$, sin importar su pasado o lo que sienta, las ideas siempre estarán ahí, aunque no significa que se arriesgue o que las ejecute en la realidad, lo cual sí forma parte del optimismo y la proactividad. Tal como Orrego (2008) lo expresaba, cualquiera que se considere emprendedor debe tener, en cierto grado, un deseo de generar ideas diferentes y allí las emociones no juegan un papel principal. Peñaherrera y Cobos (2012) abordan ampliamente la temática, dando importancia fundamental a esta variable en las personas que desean incursionar en el emprendimiento.

En cuanto a la persistencia, la investigación de Loli et al. (2009) mostró que esta se relacionaba con la experiencia laboral más que con situaciones de tipo emocional; es decir, que aquellos estudiantes que habían trabajado formal e informalmente, le daban más importancia a ser persistentes. Este asunto viene a ser cuestión de sobreponerse e intentar nuevamente, buscando soluciones alternativas; por lo tanto, se relaciona más con estilos emocionales que sean positivos.

Referente a la segunda hipótesis, los resultados correlacionales sustentan las relaciones planteadas, debido a que el estilo emocional rumiación estuvo presente en dos de las tres relaciones significativas encontradas entre las dimensiones de la variable actitud emprendedora. La actitud emprendedora, en general, está relacionada con la personalidad optimista, proactiva, persistente y creativa, por eso es de esperar que una persona que no supera los eventos del pasado, tampoco podrá tener una actitud positiva ante eventos futuros que requieren confianza

94 Emilse Durán-Aponte y Diana Arias-Gómez

Número publicado el 1 de julio del 2016 
en sí mismo y en los demás, además de reducir la ansiedad y manejar adecuadamente las expectativas y por ende, tenderá a ser pesimista y reactivo.

En cambio, la inhibición emocional puede que no obstaculice la creatividad y el desarrollo de ideas, y sí lo haga con la persistencia y el optimismo, pues se relaciona con esconder las emociones; por lo tanto, el individuo podría sentirse con ánimo, ideas y entusiasmo para emprender el negocio, solo que no desee compartir esos sentimientos con otros por temor o razones personales.

Por último, los resultados obtenidos a través del análisis de regresión múltiple confirman la tercera hipótesis parcialmente, dado que uno solo de los estilos emocionales resultó significativo estadísticamente para explicar la actitud emprendedora. Resulta comprensible, por todo lo expuesto anteriormente, que cuando se tiene una menor capacidad para superar eventos del pasado y mayor miedo a fracasar, el individuo tendrá una menor disposición a encarar de forma positiva, optimista y creativa las oportunidades de negocio que se le presenten. Tal como lo afirmaban Santos, Requero, Cancela, y Pedraza (2015), cada vez que las personas obtienen éxitos personales su autoestima va a aumentar, pero cuando se trata de fracasos esta disminuye. En este sentido, resulta importante saber cómo manejar las emociones ante nuevos retos para que no se conviertan en obstáculos para emprender, pues en el emprendimiento siempre se estará ante la incertidumbre, tropiezos y adversidades.

\section{Conclusiones}

Atendiendo a los planteamientos en las hipótesis de esta investigación, se puede decir en concreto que la muestra que participó en el estudio se relaciona con una alta orientación hacia el cambio y la innovación, y presenta un perfil caracterizado con la necesidad del desarrollo de fortalezas personales para superar la adversidad, las cuales le permitirían mantenerse exitoso en el negocio propio a futuro. Además, los resultados reflejan que una persona con alta actitud proactiva y optimista, tiene una relación negativa con los estilos emocionales inhibición y rumiación y en el caso de la creatividad e innovación, ninguna relación con dichos estilos.

En general, los resultados aquí mostrados no son desalentadores; por el contrario, parecen ser una clara posibilidad de desarrollar actitudes sólidas hacia el emprendimiento, pues se conoce en qué áreas trabajar para fortalecer la proactividad y el optimismo de los potenciales emprendedores y cómo la inhibición y la rumiación afectan estas variables. En este sentido, cuando se reconoce a una persona indecisa o confundida sobre el tema del emprendimiento, Santos et al. (2015) consideran que esta hace un uso mayor de sus recursos mentales para procesar el mensaje o la información que se le dé, debido a que está en un intento por resolver sus dudas y busca información relevante que le ayude a tomar una decisión, y allí es donde hace falta que las instituciones de educación superior fijen como norte el ofrecer información relevante que genere respuestas acertadas hacia el emprendimiento. 
Teniendo esto en cuenta y en atención al objetivo de este trabajo, que consistió en analizar la relación que existe entre la actitud emprendedora de un individuo y los estilos emocionales que posee, se realizan las siguientes apreciaciones que pueden ser claves para entender de qué manera contribuir en la formación emprendedora:

- La formulación de un plan deberá pasar por un diagnóstico de las características psicológicas de cada potencial emprendedor. Dicho examen debe basarse en el uso de instrumentos de medición debidamente adaptados y validados en la población a trabajar, como es el caso de los utilizados en el estudio (STE y CMA). Este inicio marcará el énfasis sobre el cual se deberá trabajar, toda vez que se puedan reducir las tendencias a poco socializar, el temor a compartir las emociones y el miedo a fracasar.

- El plan también deberá contemplar, adecuadamente, el desarrollo de la actitud emprendedora con el uso de estrategias adecuadas para potenciar la personalidad proactiva y optimista, y reducir los estilos emocionales inadecuados que generan pesimismo y una actitud reactiva. Por lo tanto, este plan no puede estar basado en medidas efectistas de corto plazo, delegadas en una asignatura o pocas asignaturas, pues se trata de actitudes cognitivas que si bien pueden ser entrenadas como lo afirma Seligman (2014), debe dársele el tiempo y el trato adecuado para ello. En términos generales, para Santos et al. (2015) si se logra que potenciales emprendedores presten atención a un mensaje persuasivo sobre emprender un negocio, habrá más posibilidades de generar actitudes positivas hacia el emprendimiento; por ello, al intentarles convencer de tomar este camino, valdría la pena también resaltar las consecuencias positivas de atreverse a hacerlo, sugiriendo, entre otras cosas, que de este modo se evitaría estar desempleado o forzosamente empleado en algo que no es de su agrado o donde no tiene independencia.

- En cuanto a las emociones, Durán-Aponte (2013a) sugiere que un elemento clave para la formación de emprendedores debe enfocarse en el manejo adecuado de las emociones, asumiendo de antemano que iniciar un negocio propio conlleva a un posible desgaste emocional. Por ello, esta autora recomienda basar la formación en los siguientes aspectos: perfil mesurado, manejo de la incertidumbre, eliminar los miedos ante otros, generar confianza y aclarar las consecuencias de la autosuficiencia, los cuales pueden conducir al individuo hacia un perfil de optimismo y proactividad, haciendo uso adecuado de las emociones. Salvador y Morales (2009) recomiendan realizar programas de entrenamiento para el control y el uso de las emociones, puesto que contribuyen con el desarrollo óptimo del autoempleo. Adicionalmente, para sortear los fracasos y la forma de responder emocionalmente ante ellos, Santos et al. (2015) recomiendan comenzar realizando tareas medianamente exigentes que la persona tenga una alta probabilidad de ejecutar con éxito y, de esta manera, también contribuir con mejorar la autoestima. 
- Para el caso del desarrollo de una conducta proactiva, Ares (2004) sugiere que debe enseñársele al individuo a no preocuparse tanto de dar respuestas sino de hacerse preguntas. Estas preguntas deben estar enfocadas a responderse qué estoy haciendo, cómo lo estoy haciendo, por qué lo hago, cómo me gustaría hacerlo, existe otra manera de hacerlo, cuáles cosas debería cambiar o cuáles creo que mañana no van a funcionar. De esta manera, según este autor, el individuo podrá afrontar mejor los problemas, prever consecuencias y orientarse a la innovación. Además, entre las recomendaciones para desarrollar la proactividad en potenciales emprendedores, según Santos et al. (2015), están la necesidad de detectar con qué valores de la actividad emprendedora se sienten más identificados los individuos y de esa forma, incluirlos en el mensaje que se tenga preparado para ellos; por ejemplo, si lo que más les gusta es crear un proyecto propio o lo que más les entusiasma en la posibilidad de tener autonomía familiar, autonomía económica o alcanzar un sueño, lo más aconsejable es que se les persuada de que esto es posible en la actividad emprendedora.

- En cuanto a la conducta optimista, la formación deberá tener en cuenta los planteamientos de López y García (2011), pues el optimismo favorece la relación que se establece entre las expectativas positivas y las situaciones en las que se requieren comportamientos dirigidos a conseguir objetivos difíciles, pues la persona enfrentará el desafío con más vigor y más persistencia. Por lo tanto, se debe persuadir a la persona de que le ocurrirán eventos positivos en vez de negativos y para ello, podrían disponerse de testimonios de emprendedores que al pasar por eventos riesgosos, difíciles y negativos, lograron sobreponerse y lograr el éxito.

Para finalizar, es conveniente dedicar un espacio para señalar las limitaciones del estudio y además, futuras propuestas de investigación en este contexto. En primer lugar, se puede resaltar el hecho que no se incluyeran variables de tipo contextual, debido a que todo emprendimiento se da alrededor de un conjunto de condiciones externas que motivan o desmotivan la idea de emprender. Sin embargo, el objetivo de este estudio intentaba desarrollar, más a fondo, los hallazgos de una investigación previa (Durán-Aponte \& Arias-Gómez, 2015) y aportar indicaciones claras para la formación en variables de tipo psicológicas de futuros emprendedores. En segundo lugar, no se analizaron las diferencias entre las dimensiones de actitud emprendedora y los estilos emocionales en función del género o del área de estudio, relación que definitivamente puede aportar indicios más claros sobre la naturaleza de estas variables, por lo que es conveniente sugerir para futuras investigaciones, la inclusión de análisis que indaguen sobre posibles diferencias significativas.

En tercer lugar, vale la pena mencionar que la mayoría de los estudios relacionados con el perfil de un emprendedor (Sánchez et al., 2005; Ortiz, Duque \& Camargo, 2008; Salvador, 2008; Roth \& Lacoa, 2009; Sánchez, 2010; Amorós, 2011; Moriano et al., 2012 y Mata et al., 2015) han sido realizados con 
muestras de estudiantes universitarios, pues se les considera potenciales emprendedores. Es por esto, que la muestra que participó en el estudio, si bien no estuvo conformada por emprendedores, pertenecen a un grupo que, en definitiva, tiene altas tendencias a incursionar en el autoempleo o emprendimiento; además, el hecho de ser jóvenes y estudiantes universitarios indistintamente de la carrera que cursan, les acerca más a actividades de riesgo, independientes y creativas que conducen hacia un emprendimiento a futuro. Por ello, un elemento importante que se desprende de este trabajo para futuras investigaciones, es poder diferenciar si los emprendedores reales muestran el mismo patrón en actitud emprendedora y estilos emocionales que los emprendedores potenciales.

Por último, debido a las características de la muestra y el método empleado, los resultados obtenidos en el presente estudio no son concluyentes, pero sugieren relaciones interesantes e importantes de profundizar en estudios siguientes sobre las características psicológicas y emocionales de futuros emprendedores. De esta manera, el estudio puede considerarse valioso al relacionar dos elementos clave para comprender la personalidad del futuro emprendedor y haber aplicado una técnica de análisis multivariante para encontrar relaciones significativas entre los conceptos planteados. Además, de esto se desprende que el emprendimiento es un fenómeno multicausal y se ha hecho énfasis hacia un área que hasta ahora ha sido poco considerada en los planes formativos universitarios.

Teniendo en cuenta las recomendaciones en las investigaciones previas, un plan de formación deberá considerar que el emprendedor no es solo el producto de la crianza, personalidad y factores psicológicos, es el resultado de la mezcla de elementos propios de la cultura, familia, experiencias laborales y situaciones económicas que, junto a variables como la persistencia, el optimismo, la creatividad y la proactividad, se configuran para potenciar el emprendimiento. Futuras investigaciones deberán orientarse a la configuración de modelos mixtos que permitan, a través de una robusta explicación teórica, el desarrollo de planes de formación realistas y exitosos.

Es importante agradecer a los evaluadores de este artículo, quienes aportaron sugerencias que mejoraron de forma significativa este escrito, sus críticas de tipo constructivo y formativo hicieron posible ofrecer una mejor contribución en este trabajo.

\section{Referencias}

Alda-Varas, R., Villardón-Gallego, L. \& Elexpuro-Albizuri, I. (2012). Propuesta y validación de un perfil de competencias de la persona emprendedora. Implicaciones para la formación. Electronic Journal of Research in Educational, 10 (28), 1057-1080. Recuperado de: http:// www.redalyc.org/pdf/2931/293124654006.pdf

Alvarado, O. \& Rivera, W. (2011). Universidad y emprendimiento, aportes para la formación de profesionales emprendedores. Cuadernos de Administración, 27 (45), 61-74. Recuperado de: $\underline{\text { http://www.redalyc.org/articulo.oa?id=225019868005 }}$ 
Amorós, L. (2011). El Proyecto Global Entrepreneurship Monitor (GEM): Una aproximación desde el contexto latinoamericano. Revista Latinoamericana de Administración, 46, 1-15. Recuperado de: https://www.icesi.edu.co/cdee/images/informes/colombia/REPORT GEM Colombia 2011.pdf

Ares, A. (2004). La conducta proactiva de los emprendedores. Portularia, 4, 493-498. Recuperado de: http://www.unizar.es/proactividad/articulos/articulo2.pdf

Arroyo-Vázquez,M.\&Jiménez-Sáez,F.(2008).Laincorporación delainnovaciónyelemprendedurismoen laEducaciónSuperior:unaformación defuturo.(Trabajo presentadoalVCongresolberoamericano de Docencia Universitaria). Recuperado de: http://digital.csic.es/bitstream/10261/17160/1/ AC235 1 526-ARROYO\%20JIMENEZ\%20Docencia\%20Universitaria.pdf

Bernal, A. (2014). Competencia emprendedora e identidad personal. Una investigación exploratoria con estudiantes de Educación Secundaria obligatoria. Revista de Educación, 363, 384-411. Recuperado de: http://www.revistaeducacion.mec.es/doi/363 192.pdf

Cordero, J., Astudillo, S., Delgado, J., Amón. O. \& Carpio, X. (2011). Análisis de los factores que influyen el emprendimiento y la sostenibilidad de las empresas del área urbana de la ciudad de Cuenca, Ecuador. MASKANA, 2 (2), 27-37. Recuperado de: http://dspace.ucuenca.edu. ec/handle/123456789/5412

Durán-Aponte, E. (2013a). Análisis del perfil emprendedor en estudiantes universitarios del área administrativa: aportes a la formación gerencial emprendedora. (V Congreso de Gerencia en América Latina: Tendencias gerenciales desde una visión crítica). Venezuela: Universidad del Zulia.

Durán-Aponte, E. (2013b). Distinción entre actitud emprendedora y autoeficacia emprendedora: Validez y confiabilidad en estudiantes universitarios. Educación y futuro digital, (7), 59-69. Recuperado de: http://www.cesdonbosco.com/documentos/revistaeyfd/EYFD 7.pdf

Durán-Aponte, E. \& Arias-Gómez, D. (2015). Intención emprendedora en estudiantes universitarios: integración de factores cognitivos y socio-personales. Revista Colombiana de Ciencias Sociales, 6 (2), 320-340. Recuperado de: http://www.funlam.edu.co/revistas/ index.php/RCCS/article/view/1528

Espíritu, R. (2011). Análisis de la intención emprendedora en estudiantes universitarios a través de los rasgos de personalidad. Multiciencias, 11 (1), 65-75. Recuperado de: http://www. redalyc.org/articulo.oa?id $=90418851009$

Flores, M. \& Barberá, J. (2013). Evaluación del impacto de la educación superior en la iniciativa emprendedora. Historia y Comunicación Social, 18, 377-386. Recuperado de: file:///C:/ Users/equipo\%201/Downloads/44256-68225-3-PB\%20(1).pdf

Gardner, H. (1995). Inteligencias múltiples: La teoría en la práctica. Barcelona: Paidós. 
Gardner, H. (2000). Intelligence reframed: Multiple intelligences for the 21st Century. New York: Basic Books.

Goleman, D. (1995). Inteligencia emocional. España: Editorial Kairos.

Goleman, D. (1998). Whats makes a Leader? Estados Unidos: Harvard Business Review.

González, N. \& Valdez, J. (2012). Optimismo-pesimismo y resiliencia en adolescentes de una universidad pública. CIENCIA ergo sum, 19 (3), 207 - 214. Recuperado de: http://www. redalyc.org/articulo.oa?id $=10423895002$

Guarino, L. (2011). Adaptación y validación de la versión hispana del Cuestionario de Estilo Emocional. Universitas Psychologica, 10 (1), 197-209. Recuperado de: file:///C:/Users/ equipo\%201/Downloads/303-4118-1-PB.pdf

Kerlinger, F. \& Lee, H. (2002). Investigación del comportamiento: Métodos de investigación en Ciencias Sociales. México: McGraw-Hill.

Loli, A., Del Carpio, J. \& La Jara, E. (2009). El emprendimiento en los estudiantes de la UNMSM y su relación con algunas variables sociodemográficas. Revista de Investigación en Psicología, 12 (1), 111-130. Recuperado de: http://revistasinvestigacion.unmsm.edu.pe/index.php/ psico/article/view/3786

López, J. \& García, J. (2011). Optimismo, pesimismo y realismo disposicional en emprendedores potenciales de base tecnológica. Psicothema, 23 (4), 611-616. Recuperado de: http://www. psicothema.com/pdf/3930.pdf

Marulanda, J., Correa, G. \& Mejía, L. (2009). Emprendimiento: Visiones desde las teorías del comportamiento humano. Revista Escuela de Administración de Negocios, 66, 153-168. Recuperado de: $\underline{\text { http://www.redalyc.org/articulo.oa?id=20620269008 }}$

Martínez, F. \& Carmona, G. (2009). Aproximación al concepto de "competencias emprendedoras": Valor social e implicaciones educativas. Revista Iberoamericana sobre Calidad, Eficacia y Cambio en Educación, 7 (3), 82-98. Recuperado de: http://www.redalyc.org/articulo. oa?id=55114063007

Mata, A., Medina, F., Martínez, E., Alcocer, R., Castillo, A. \& Vázquez, V. (2015). Competencias claves para el emprendimiento. La perspectiva de los estudiantes. Universo de la Tecnológica, 7 (20), 13-15. Recuperado de: http://132.248.9.34/hevila/Universodelatecnologica/2014-2015/ no20/2.pdf

Mayer, J., Caruso, D. \& Salovey, P. (1999). Emotional intelligence meets traditional standards for an intelligence. Intelligence, 27 (4), 267-298. Recuperado de: http:// www.unh.edu/emotional intelligence/El\%20Assets/Reprints...El\%20Proper/ El1999MayerCarusoSaloveylntelligence.pdf 
Mayer, J. \& Salovey, P. (1997). What is emotional intelligence? En: P. Salovey y D. J. Sluyter (Eds), Emotional development and emotional intelligence: Educational implications, pp. 3-34. New York: Harper Collins.

Merino, M. \& Vargas, D. (2011). Evaluación comparativa del potencial emprendedor de Latinoamérica: una perspectiva multinivel. Academia Revista Latinoamericana de Administración, 46, 38-54. Recuperado de: http://www.redalyc.org/articulo. oa?id=71617238004

Moriano, J., Palací, F. \& Morales, J. (2006). Adaptación y validación en España de la escala de autoeficacia emprendedora. Revista de Psicología Social, 21 (1), 51-64. Recuperado de: http:// www2.uned.es/dpto-psicologia-social-y-organizaciones/paginas/profesores/moriano/ copiaseguridad/J.A.Moriano archivos/21 1\%5B1\%5D.\%20MORIANO\%20ET\%20AL.pdf

Moriano, J., Topa, G., Morelo, F., Entenza, A. \& Lévy-Mangin, L. (2012). Autoeficacia para el liderazgo emprendedor. Adaptación y validación de la escala CESE en España. Anales de psicología, 28 (1), 171-179. Recuperado de: http://www.redalyc.org/articulo.oa?id=16723161019

Orrego, C. (2008). La dimensión humana del emprendimiento. Revista Ciencias Estratégicas, 16 (20), 225-235. Recuperado de: https://revistas.upb.edu.co/index.php/cienciasestrategicas/ article/view/582

Ortiz, C., Duque, Y. \& Camargo, D. (2008). Una revisión a la investigación en emprendimiento femenino. Investigación y Reflexión Revista Facultad de Ciencias Económicas, 16 (1), 85-104. Recuperado de: http://www.redalyc.org/articulo.oa?id=90916107

Peñaherrera, M. \& Cobos, F. (2012). La creatividad y el emprendimiento en tiempos de crisis. Revista Iberoamericana sobre Calidad, Eficacia y Cambio en Educación, 10 (2), 238-247. Recuperado de: http://www.rinace.net/reice/numeros/arts/vol10num2/art15.pdf

Pihkala, T. \&Vesalainen, J. (2000). Barriers to entrepreneurship-educational opportunities. (Trabajo presentado al IntEnt 2000). Finland: Tampere University of Technology.

Roth, E. \& Lacoa, D. (2009). Análisis psicológico del emprendimiento en estudiantes universitarios: Medición, relaciones y predicción. Ajayu, 7 (1), 1-38. Recuperado de: http://www.scielo. org.bo/scielo.php?script=sci arttext\&pid=S2077-21612009000100005

Salazar-Carvajal, P., Herrera-Sánchez, I., Rueda-Méndez, S. \& León-Rubio, J. (2014). El efecto de la conservación de recursos sobre la intención emprendedora en el contexto de crisis económica: el rol moderador de la autoeficacia y la creatividad. Anales de Psicología, 30 (2), 549-559. Recuperado de: http://scielo.isciii.es/scielo.php?script=sci arttext\&pid $=$ S0212-97282014000200018

Salvador, C. (2008). Impacto de la inteligencia emocional percibida en la autoeficacia emprendedora. Boletín de Psicología, 92, 65-80. Recuperado de: http://www.territoriochile. 
$\mathrm{cl} /$ modulo/web/competencias emocionales/inteligencia\%20emocional\%20y\%20 eficacia\%20emprendedora.pdf

Salvador, C. (2009). Ecuaciones estructurales como modelos predictivos de la autoeficacia emprendedora en una muestra de jóvenes mexicanos y españoles. Apuntes de Psicología, 27 (1), 65-78. Recuperado de: http://www.cop.es/delegaci/andocci/files/contenidos/ VOL.\%2027 1 2009/vol.\%2027 1 4.pdf

Salvador, C. \& Morales, J. (2009). Fundamentos psicológicos de la autoeficacia emprendedora en jóvenes mexicanos. Alternativas en Psicología, 14 (20), 35-47. Recuperado de: http:// pepsic.bvsalud.org/scielo.php?script=sci arttext\&pid=S1405-339X2009000100004

Sánchez G., J. C. (2010). Evaluación de la personalidad emprendedora: validez factorial del Cuestionario de Orientación Emprendedora (COE). Revista Latinoamericana de Psicología, 42 (1), 41-52. Recuperado de: http://www.redalyc.org/articulo.oa?id=80515880004

Sánchez, J., Caggiano, V. \& Hernández, B. (2011). Competencias emprendedoras en la educación universitaria. International Journal of Developmental and Educational Psychology, 3 (1), 19-28. Recuperado de: http://infad.eu/RevistaINFAD/2011/n1/volumen3/INFAD 010323 19-28.pdf

Sánchez, J., Lanero, A. \& Yurrebaso, A. (2005). Variables determinantes de la intención emprendedora en el contexto Universitario. Revista de Psicología Social Aplicada, 15 (1), 37-60. Recuperado de: http://psi.usal.es/emprendedores/documentos/Sanchez05.pdf

Santos, D., Requero, B., Cancela, A. \& Pedraza, A. (2015). Juventud proactiva: Cómo crear unas actitudes fuertes y positivas hacia el emprendimiento. Revista de Estudios de Juventud, 15 (107), 125-147. Recuperado de: http://www.injuve.es/sites/default/files/2015/19/ publicaciones/Documentos\%208\%20Juventud\%20proactiva.pdf

Shapero, A. \& Sokol, L. (1982). The social dimensions of entrepreneurship. En: C. Kent, D. Sexton \& K. H. Vesper (Eds.), Encyclopaedia of Entrepreneurship, 72-90. Englewood Cliffs, NJ: Prentice Hall.

Seligman, M. (2014). Aprenda optimismo: Haga de la vida una experiencia gratificante. España: DEBOLSILLO. 